\title{
EFEITO do ÁCIDO ACEILSALCÍlLICO NA PERForMANCE REPRODUTIVA E NA PROLE DE RATAS WISTAR
}

\author{
Débora Cristina Damasceno*, Gustavo tadeu Volpato, Osmar Clayton Person, Alessandra Yoshida, \\ Marilza Vieira Cunha Rudge, Iracema de Mattos Paranhos Calderon \\ Trabalho realizado na Faculdade Estadual de Medicina de Marilia, Marilia, São Paulo
}

Resumo - 0 objetivo deste trabalho foi realizar um estudo randomizado, controlado e "cego" para investigar se a dose terapêutica de ácido acetilsalićílico (AAS), usado por mulheres grávidas, pode causar efeito tóxico ao embrião ou anormalidades congênitas nos animais de experimentação.

Métodos. Foi confirmada a prenhez das fêmeas através da observação da presença de espermatozóides no esfregaço vaginal ( ${ }^{\circ}$ dia de prenhez - GDI). As ratas acasaladas foram colocadas em gaiolas individualizadas e divididas aleatoriamente para compor os grupos experimentais. Os animais receberam $50 \mathrm{mg} / \mathrm{kg} / \mathrm{dia}$ de AAS, por via oral (gavage), durante dois períodos diferentes de prenhez. Um grupo de ratas $(n=\mathrm{II})$ recebeu aspirina do $1^{\circ}$ ao $4^{\circ}$ dia de prenhez (antes de implantação embrionária) para avaliação dos blastocistos, e outro grupo recebeu aspirina do $6^{\circ}$ ao $15^{\circ}$ dia (período de organogênese) para avaliação fetal. Os grupos con- trole $(n=12)$ receberam água destilada com mesmo volume e durante os mesmos períodos, semelhante aos respectivos grupos experimentais.

Resultados e Conclusão. 0 tratamento de ratas prenhes com AAS, de acordo com dose terapêutica mínima usada por mulheres, não causou efeitos embriotóxicos ou malformações maiores, mas foi responsável pelo aumento das taxas de fetos com dilatação de ureter. Pode ser verificada uma divergência muito grande entre os dados obtidos experimentalmente e em humano, mas não há ainda uma evidência conclusiva dos efeitos adversos em humanos. Existe uma dose de perigo potencial, portanto o uso indiscriminado de ácido de acetilsalicílico (aspirina) é contra-indicado.

UnITERMos: Aspirina. Acido acetilsalicílico. Gravidez. Pré-implantação. Ratos.

\section{INTRODUÇÃo}

As drogas antiinflamatórias não-esteroidais (FAINES) ganharam popularidade no final dos anos de 1970'. Os salicilatos sintéticos são extensivamente usados como drogas de prescrição devido as suas propriedades analgésica, antipirética e antiinflamatória. Aspirina (ácido acetilsalicílico) inibe a síntese de prostaglandinas (PGs) por acetilação irreversível deácido graxo, inativando a enzima ciclooxigenase ${ }^{2}$.

Com o aumento do uso indiscriminado de medicamentos e com a crescente produção de substâncias químicas, surgiu a suspeita de um efeito comum na gravidez após a exposição a agentes químicos que poderia ter um papel na etiologia das malformações fetais ${ }^{3}$. Kimmel et al. ${ }^{4}$ verificaram que a aspirina é um agente causador de teratogênese. Da mesma forma, outros autores observaram que 0 ácido salićlico, produto da hidrólise da aspirina, seria oagente teratogênico responsável ${ }^{5}$.

\footnotetext{
*Correspondência:

Disciplina de Embriologia - FAMEMA

Av. Monte Carmelo, 800

CEP. 175 19-030-Marilia-São Paulo-Brasil

E-mail: decaito@bol.com.br
}

Embora o uso de aspirina seja muito utilizado por mulheres durante a gestação, mesmo sem prescrição médica, seus efeitos no feto permanecem controversos. Espiridião et al. ${ }^{6}$, administrando AAS em diferentes doses a ratas do $5^{\circ}$ ao $20^{\circ}$ dia de prenhez, verificaram diminuição do número de fetos vivos, redução do peso fetal e placentário e queda do ganho de peso materno. Quando ratas prenhes foram tratadas com 200 e $500 \mathrm{mg}$ de AAS/kg de peso corpóreo, o estudo mostrou alterações morfológicas no feto e na placen$\mathrm{ta}^{7}$. Com a finalidade de avaliar a eficácia da aspirina $(100 \mathrm{mg})$ na prevenção de retardo de crescimento intra-uterino ( $\mathrm{RCIU}$ ) em gravidez de risco, foi realizado um ensaio clínico experimental duplo cego, placebo-controlado, e foi verificado que a incidência de RCIU foi nula no grupo de mulheres que receberam aspirina ${ }^{8}$.

O objetivo deste trabalho foi realizar um estudo randomizado, controlado e "cego" para investigar se a dose terapêutica de ácido acetilsalicílico (AAS), usado por mulheres grávidas, pode causar efeito tóxico ao embrião ou anormalidades congênitas nos animais de experimentação.

\section{MÉTodos}

Protocolo: Ratas Wistar albinas (10-12 semanas de idade) foram obtidas do Biotério Central da Unesp-Campus de Botucatu (São Paulo, Brasil). Foram mantidos quatro animais por gaiola em ambiente com condições controladas (temperatura $22 \pm 2{ }^{\circ} \mathrm{C}$, I2 $\mathrm{hr}$ claro/ 12 ciclo escuro) com acesso livre para água e ração. Após 10 dias de aclimatização em condições padrão, foi realizado período de acasalamento.

Para o experimento, dois comprimidos de ácido acetilsalicílico (AAS) foram diluídos em água destilada. Adose administrada foi $50 \mathrm{mg} /$ $\mathrm{kg}$, semelhante ao efeito farmacológico mínimo produzido em humanos (uma dose diária de $3000 \mathrm{mg}$ de AAS para um indivíduo pesando em torno de $60 \mathrm{~kg}$ ).

Grupos Experimentais: Os animais receberam AAS por via oral (gavage) durante dois períodos diferentes de prenhez. Um grupo de ratas $(n=I I)$ recebeu aspirina do $1^{\circ}$ ao $4^{\circ}$ dia de prenhez (antes de implantação embrionária) para avaliação dos blastocistos e outro grupo recebeu aspirina do $6^{\circ}$ ao $15^{\circ}$ dia (período de organogênese) para avaliação fetal. 
Os grupos-controle $(n=12)$ receberam água destilada com mesmo volume e durante os mesmos períodos, semelhante aos respectivos grupos experimentais.

Avaliação dos Blastocistos: Para o acasalamento, foram colocadas duas ratas na presença de um macho (2:I). Foi confirmada prenhez através da visualização de espermatozóides no esfregaço vaginal, este foi considerado ${ }^{\circ}$ dia de prenhez (GDI) ${ }^{9}$. As fêmeas acasaladas foram distribuídas aleatoriamente nos diferentes grupos experimentais e colocadas em gaiolas individuais. Durante a prenhez, os animais foram observados diariamente quanto à taxa de mortalidade e qualquer mudança de comportamento. Foram verificados os pesos dos animais em GDI e GD5. Em GD5, as ratas foram mortas por inalação excessiva de éter. Em seguida, foi realizada laparotomia para avaliação dos blastocistos e o investigador "cego" desconhecia a que grupo pertencia o referido animal. Os cornos uterinos foram expostos, cada um foi lavado com I ml de solução fisiológica para coleta dos blastocistos em um vidro de relógio. Caso houvesse erro técnico durante a coleta, os cornos uterinos eram excluídos. Os embriões (blastocistos) coletados foram examinados sob microscópio de contraste de fase para classificação e observação de anormalidades morfológicas. Os embriões foram classificados como anômalo quando apresentaram alterações morfológicas como: desarranjo ou nenhuma densidade de suas células (blastômeros) ou forma irregular da massa celular do embrião quando comparados ao grupo controle ${ }^{10}$.

Estudo de Teratogenicidade em Ratos: Durante a prenhez, foi observado diariamente taxa de mortalidade e qualquer mudança de comportamento das ratas. Foram observados pesos dos animais nos dias I, 7, I4 e 21 de prenhez. Em GD2I, as ratas foram mortas por inalação excessiva de éter e foi realizada laparotomia para avaliação do desempenho reprodutivo pelo investigador "cego". Após exposição dos cornos uterinos, foi realizada a contagem de: número de corpos lúteos, de implantação, de fetos mortos, de reabsorção embrionária (aborto) e de fetos vivos. Oíndice placentário foi calculado pela relação entre peso da placenta e peso corpóreo do feto. Os pesos corpóreos dos recém-nascidos foram classificados como pequeno (PIP), adequado (AIP) egrande (GIP) para idade de prenhez, de

Tabela I - Avaliação dos blastocistos no $5^{\circ}$ dia de prenhez após tratamento com ácido acetilsalicílico

\begin{tabular}{|c|c|c|}
\hline & Controle & $\begin{array}{c}\text { AAS } \\
(50 \mathrm{mg} / \mathrm{Kg})\end{array}$ \\
\hline Ratasacasaladas & II & II \\
\hline Ratas prenhes & II & II \\
\hline Fertilidade(\%)a & 100 & 100 \\
\hline Ratas prenhes sobreviventes $(\%)$ & 100 & 100 \\
\hline $\mathrm{N}^{\circ}$ Corpos Lúteos & 168.0 & 143.0 \\
\hline (média $\pm d p)$ & $15.27 \pm 4.24$ & $13.00 \pm 3.13$ \\
\hline NoBlastocistosobservados & 140.0 & 114.0 \\
\hline (média $\pm d p)$ & $12.72 \pm 3.44$ & $10.36 \pm 1.67$ \\
\hline Comalteraçõesmorfológicas & $14 / 140$ & $7 / 114$ \\
\hline Com retardo de desenvolvimento & $|7 /| 40$ & $5 / 114$ \\
\hline Ganho de peso materno (g) & & \\
\hline (média $\pm d p)$ & $19.63 \pm 6.18$ & $12.72 \pm 6.40 *$ \\
\hline
\end{tabular}

${ }^{a}\left(N^{\circ}\right.$ Ratas prenhes $/ N^{\circ}$ Ratas acasaladas $) \times 100$

* $p<0.05$-Teste de Mann-Whitney comparado ao grupo controle

acordo com o método proposto por Calderon". Os sítios de implantação foram determinados pelo método de Salewski ${ }^{12}$. 0 número total de corpos lúteos de cada ovário também foi verificado. A taxa de perda préimplantação foi calculada como: número de corpos lúteos - número de implantação x 100/ número de corpos lúteos. A taxa de perda pós-implantação foi calculada como: número de implantação - número de fetos vivos x $100 /$ número de implantação ${ }^{\prime 3}$.

Após exame externo, metade de cada ninhada foi examinada quanto à presença de malformações e/ou anomalias viscerais de acordo com o método de Wilson ${ }^{14}$. A outra metade dos fetos foi eviscerada, diafanizada e corada com Alizarina, segundo a técnica de Staples eSchnell ${ }^{15}$ para análise de anormalidades esqueléticas. $O$ grau de ossificação foi avaliado segundo método proposto por Aliverti et al. ${ }^{16}$.

Análise Estatística: Cada ninhada foi considerada como uma unidade experimental e os dados foram expressos como média de fetos por ninhada. Peso materno, placentário e peso dos fetos foram comparados por Análise de Variância. Foi realizado teste de MannWhitney para comparar número de corpos lúteos, de implantação, de reabsorção embrionária, de fetos mortos e vivos ${ }^{17}$. A porcentagem de fetos afetados em cada ninhada, computada para cada tipo de anormalidade, e a freqüência de ninhada com fetos malformados e anômalos foram analisadas através do teste do qui-quadrado ${ }^{18}$. 0 nível de significância estatística foi considerado $\mathrm{P}<0.05$.

\section{Resultados}

Avaliação dos Blastocistos: O tratamento de ratas com AAS durante período de préimplantação promoveu diminuição do ganho de peso corpóreo. Foi verificado que o número de blastocistos coletados e observados não diferiu entre os grupos controle e tratado. 0 número de embriões com alterações morfológicas foi semelhante nos grupos experimentais (Tabela I).

Estudo de Teratogenicidade em Ratos: No dia de concepção, o peso corpóreo das ratas do grupo tratado tendeu a ser menor que no grupo controle (Tabela 2). $O$ tratamento com AAS não mostrou ter qualquer efeito negativo sobre a saúde do animal, sobre 0 consumo de ração ou ganho de peso. Porém, o tratamento com ácido acetilsalicílico diminuiu a taxa de prenhez de 100\% (grupo controle) para 92,3\% (AAS) (Tabela 2).

O número de implantações por ninhada foi semelhante nos dois grupos (Tabela 2). 0 número total de reabsorção tendeu a ser mais alto que o grupo de controle, mas nenhum efeito consistente do AAS pôde ser identificado (Tabela 2). A porcentagem de perda de embrião antes da implantação aumentou de $4,73 \%$ (grupo controle) para I8, II\% (AAS). Após o processo de implantação embrionária, a porcentagem de perda de embrião aumentou também de $5,10 \%$ para $12,24 \%$, mas não 
Tabela 2 - Efeito do ácido acetilsalićlico sobre a performance reprodutiva de ratas

\begin{tabular}{|c|c|c|}
\hline & Controle & $\begin{array}{c}\text { AAS } \\
(50 \mathrm{mg} / \mathrm{Kg})\end{array}$ \\
\hline Ratasacasaladas & 12 & 13 \\
\hline Ratas prenhes atermo & 12 & 13 \\
\hline Fertilidade(\%)a & 100.0 & 90.0 \\
\hline \multicolumn{3}{|l|}{ Ganhode peso materno(g) } \\
\hline Dia $21^{\circ}-1^{\circ}($ média $\pm d p)$ & $103.00 \pm 24.22$ & $95.07 \pm 36.42$ \\
\hline Dia $21^{\circ}-1^{\circ}($ média $\pm d p)($ sem mesinha $)$ & $408.08 \pm 21.31$ & $39.17 \pm 15.36$ \\
\hline №Implantação & 142.0 & 149.0 \\
\hline (média $\pm \mathrm{dp}$ ) & $11.83 \pm 1.58$ & $11.46 \pm 3.15$ \\
\hline NNFetos vivos & 135.0 & 140.0 \\
\hline (média $\pm d p)$ & $11.25 \pm 1.81$ & $10.77 \pm 3.70$ \\
\hline №Reabsorção(Salewski +) & 7.0 & 9.0 \\
\hline PerdaPré-implantação(\%) & 4.73 & 18.11 \\
\hline PerdaPós-implantacăo(\%) & 5.10 & 12.24 \\
\hline
\end{tabular}

${ }^{a}\left(N^{\circ}\right.$ Ratas prenhes $/ \mathrm{N}^{\circ}$ Ratas acasaladas $) \times 100$

Tabela 3 - Peso do feto e da placenta no estudo teratogênico de ratas

\begin{tabular}{lcc}
\hline & Controle & AAS \\
& $(\mathbf{5 0} \mathbf{~ m g} / \mathbf{K g})$ \\
\cline { 2 - 3 } & $(\mathrm{n}=135)$ & $(\mathrm{n}=140)$ \\
Pesofetal $(\mathrm{g})($ média $\pm \mathrm{dp})$ & $3.85 \pm 0.22$ & $3.85 \pm 0.29$ \\
Pesoplacental $(\mathrm{g})($ média $\pm \mathrm{dp})$ & $0.53 \pm 0.09$ & $0.59 \pm 0.12$ \\
Índiceplacentenário & $0.14 \pm 0.02$ & $0.15 \pm 0.03$ \\
\hline
\end{tabular}

Tabela 4 - Classificação do peso corpóreo fetal no estudo teratogênico de ratas

\begin{tabular}{lcc}
\hline & & AAS \\
& Controle & $(\mathbf{5 0} \mathbf{~ m g} / \mathbf{K g})$ \\
\cline { 2 - 3 } & $(\mathrm{n}=135)$ & $(\mathrm{n}=140)$ \\
$\mathrm{PIP}(\%)$ & 20.6 & 25.7 \\
$\operatorname{AP}(\%)$ & 56.6 & 45.7 \\
$\mathrm{GIP}(\%)$ & 22.8 & 28.6 \\
\hline
\end{tabular}

foi observada nenhuma diferença estatística entre essas porcentagens (Tabela 2).

O número de fetos de ratas tratadas com AAS foi semelhante ao grupo controle. O tratamento com AAS não afetou o peso fetal (Tabela 3) e sua classificação (Tabela 4), que seguiu uma distribuição normal. $O$ peso das placentas não mostrou diferença do grupo controle. Oíndice placentário foi semelhante entre os grupos (Tabela 3).

Oexame morfológico externo foi realizado em 275 fetos. Durante análise de esqueleto do grupo controle, uma ninhada (setefetos) foi descartada devido a erro técnico durante a preparação. Não foram observadas malformações esqueléticas ou outros efeitos adversos nos fetos de ratas que receberam a dose de 50 $\mathrm{mg}$ de AAS/kg; apenas um feto do grupo controle apresentou costela ondulada. Os fetos a termo apresentaram grau de desenvolvimento semelhante, conforme demonstrado pela análise do número de centros de ossificação e através de peso fetal. Quando as mães foram expostas ao tratamento com AAS, a freqüência de fetos com malformação maior não diferiu do controle $(p>0.05)$, porém foi observado aumento significativo de recém-nascidos com dilatação de uretér, considerada uma anomalia visceral (Tabela 5).

\section{Discussão}

Aspirina (ácido acetilsalicílico) é rapidamente absorvida no estômago e no intestino superior. Em geral, $80 \%-100 \%$ de aspirina sólida (via oral) é absorvida. A taxa geralmente é mais rápida com soluções aquosas. Aspirina é então hidrolisada a ácido salicílico por esterases intestinais e hepáticas ${ }^{19}$. Foi demonstrado que a aspirina atravessa a membrana placentária rapidamente em ratos e camundongos ${ }^{20}$. Esses destinos metabólicos da aspirina têm sido considerados os mesmos para os seres humanos ${ }^{21}$.

Muitos fatores contribuem indubitavelmente para os resultados discrepantes, inclusive o período de uso de droga, a dose empregada, o tipo de análise estatística utilizado e como os fatores de confusão são controlados $^{22}$. Em vista do longo e extenso uso de salicilatos, tem sido sugerido que eles não têm nenhum potencial teratogênico demonstrável no humano ${ }^{21}$. Entretanto, pesquisas em animais de laboratório têm mostrado que a aspirina é teratogênica em altas doses, sendo o período crítico para malformações em ratos do $9^{\circ}$ ao $11^{\circ}$ dia de prenhez ${ }^{22}$. O presente estudo foi realizado com o objetivo de avaliar se o tratamento com AAS causa efeitos adversos em fetos de ratas utilizando-se dose terapêutica, ao contrário dos trabalhos realizados anteriormente que utilizaram doses elevadas.

Nossos resultados mostraram que a administração oral de AAS não causou morte embrionária, não reduziu o peso fetal, nem aumentou a taxa malformações maiores. Os resultados encontrados não confirmam os dados de Espiridião et al. ${ }^{6}$, que observou redução no número e pesos dos fetos quando as ratas foram expostas a 100 e $400 \mathrm{mg}$ de AAS/kg de peso por dia. Foi demonstrado também, em nosso trabalho, que AAS não afetou a performance reprodutiva das ratas. Foram observados alguns casos esporádicos de $14^{\mathrm{a}}$ costela nos recém-nascidos de mãe-controle e que receberam $50 \mathrm{mg} / \mathrm{kg}$ de AAS, os quais foram considerados espontâneos e não relacionados com o tratamento. As costelas supranumerárias são consideradas ocorrência comum nos estudos teratogênicos de ratos e são conside- 
Tabela 5 - Incidência de malformação e anomalia externa, esquelética e visceral nos fetos de ratas

\begin{tabular}{|c|c|c|}
\hline & Controle & $\begin{array}{c}\text { AAS } \\
(50 \mathrm{mg} / \mathrm{Kg})\end{array}$ \\
\hline \multicolumn{3}{|l|}{ MALFORMAÇÃOEXTERNA } \\
\hline FetosAfetados & 0/135 & $0 / 140$ \\
\hline Ninhadas Afetadas & $0 / 12$ & $0 / 12$ \\
\hline \multicolumn{3}{|l|}{ ANOMALIAEXTERNA } \\
\hline FetosAfetados & $1 / 62$ & $0 / 73$ \\
\hline Ninhadas Afetadas & $1 / 11$ & $0 / 12$ \\
\hline \multicolumn{3}{|l|}{ ANOMALIAESQUELÉTICA } \\
\hline FetosAfetados & $37 / 62$ & $38 / 73$ \\
\hline Ninhadas Afetadas & $\mid 1 / I 1$ & $10 / 12$ \\
\hline $14^{\mathrm{a}}$ costela & 10/62 & $12 / 73$ \\
\hline \multicolumn{3}{|l|}{ MALFORMAÇÃOVISCERAL } \\
\hline FetosAfetados & $21 / 66$ & $28 / 67$ \\
\hline Ninhadas Afetadas & $7 / 12$ & $9 / 12$ \\
\hline Hidronefrose & $21 / 66$ & $28 / 67$ \\
\hline \multicolumn{3}{|l|}{ ANOMALIAVISCERAL } \\
\hline FetosAfetados & $16 / 66$ & $32 / 67 *$ \\
\hline Ninhadas Afetadas & $07 / 12$ & $11 / 12$ \\
\hline Dilataçãode uretér & $16 / 66$ & $31 / 67 *$ \\
\hline \multicolumn{3}{|l|}{ CENTRODEOSSIFICAÇÃO } \\
\hline (média $\pm d p)$ & $31,24 \pm 1,66$ & $34,22 \pm 1,66$ \\
\hline
\end{tabular}

${ }^{*} p<0.05$-Teste qui-quadrado comparado ao grupo controle

radas como indicativo de teratogenicidade por alguns autores. No entanto, não são considerados tão indicativos por outros investigadores. Como um evento de teratogênese é, por definição, uma mudança permanente, seria necessário um estudo mais profundo para acompanhar o desenvolvimento das costelasextras durante a fase adulta ${ }^{23}$.

Estudos concluíram que a ação teratogênica dos componentes de salicilatos ocorre por fatores metabólicos mediados maternalmente, porém outros métodos têm mostrado que essa classe de droga tem um efeito direto no embrião de rato na ausência de qualquer influência materna ${ }^{24}$. Da mesma forma, autores verificaram que ratas prenhes tratadas por via oral com 500 ou $1000 \mathrm{mg} / \mathrm{kg}$ de aspirina no $3^{\circ}$ dia de prenhez não apresentaram malformações significativas nos fetos ${ }^{25}$. Entretanto, quando ratas prenhes receberam aspirina intragastricamente $(250,500$ e $1000 \mathrm{mg} / \mathrm{kg})$ no $3^{\circ}$ dia, foram induzidas malformações fetais pelo tratamento, antes de implantação embrionária, de forma dose-dependente ${ }^{26}$.

Os roedores são mais resistentes que 0 humano a muitas drogas ${ }^{27}$. 0 rim tem um papel muito importante na excreção de medicamentos e seus metabólitos, muitos dos quais provocam alterações da função renal ou dano de suas diversas estruturas (glomérulos, túbulos e interstício). As alterações produzidas podem ser agudas e reversíveis ou crônicas ${ }^{28}$. De acordo com nossos resultados, o tratamento com AAS interferiu no desenvolvimento do sistema urinário. A baixa dose de AAS foi capaz de induzir dilatação de ureter em aproximadamente $46 \%$ dos recém-nascidos. A dilatação de ureter grave é reconhecida facilmente, porque os ureteres dilatados estão moldados em "S" ou retorcidos, completamente transparentes e, então, facilmente identificáveis, como visto neste estudo. Além disso, a dilatação uretérica pode ou não estar associada à hidronefrose congênita e a quaisquer alterações pélvicas, podendo ser considerada transitória em roedores ${ }^{29}$. De acordo com dados de literatura, foi verificado que $10 \%$ a $20 \%$ dos recém-nascidos de ratas, pertencentes ao grupo controle, apresentam este tipo de anomalia visceral9 ${ }^{9,0,30}$. Apesar da taxa aumentada de fetos com hidronefrose no grupo experimental (42\%), isto não foi relacionado ao tratamento com AAS porque as ninhadas-controle também apresentaram freqüência elevada de fetos com hidronefrose (32\%) neste estudo, sugerindo que um au- mento no número de ninhadas poderia favorecer elevação na incidência desta malformação visceral em função do tratamento com ácido acetilsalicílico.

\section{Conclusão}

O modelo experimental permitiu avaliar os blastocistos e as anormalidades fetais após tratamento com AAS. O tratamento comAAS, na dose terapêutica mínima usada por mulheres, não causou efeito embriotóxico ou malformações maiores nos animais experimentais, mas foi responsável pelo aumento na taxa de recém-nascidos que apresentaram dilatação de ureter. Tem sido verificada uma divergência muito grande entre os dados obtidos experimentalmente e em humano, não havendo ainda uma evidência conclusiva sobre os efeitos adversos em humanos. Existe uma dose de perigo potencial, portanto 0 uso indiscriminado de ácido acetilsalićlico (aspirina) é contra-indicado.

\section{SUMMARY}

EFFeCt OF ACETYLSAlicylic ACID ON THE REPRODUCTIVE PERFORMANCE AND ON OFFSPRING FROM WISTAR RATS

The aim of this paper was to perform a randomized, controlled and blinded study to investigate if a therapeutic dose of acetylsalicylic acid (ASA), taken by pregnant women, may also cause embryotoxic or congenital abnormalities on experimental animal.

METHODS: Females were confirmed to have mated by observations of sperm in a vaginal smear. The day on which spermatozoa were found in the vaginal smear was considered as day I of gestation (GDI). After randomization, mated females were assigned to experimental groups and individually caged, were given $50 \mathrm{mg} /$ $\mathrm{kg} /$ day of acetylsalicylic acid, by needle gavage once daily, during two different periods of pregnancy. One group of dams $(n=I I)$ received aspirin from day I to 4 of pregnancy (before embryonic implantation) for evaluation of the blastocysts, and another group received aspirin from day 6 to 15 of pregnancy (organogenic period) for fetal evaluation. Control groups $(n=12)$ received distilled water in same volume and during same periods as their respective experimental groups.

Results and Conclusion: The treatment of the dams with ASA, according to minimal 
therapeutic dose used for humans, did not cause embryotoxic or major malformations on experimental animal but was responsible for rate increased of fetuses presenting ureteric dilatation. After analysis of the data, it appears that, although direct conclusive evidence of adverse effects in humans is lacking, a potential hazard dose exists and thus the indiscriminate use of acetylsalicylic acid (aspirin) is contraindicated. [Rev Assoc Med Bras 2002; 48(4): 312-6]

KEY WORDS: Aspirin. Acetylsalicylic acid. Pregnancy. Preimplantation. Rat.

\section{RefERÊNCIAS}

I. Schoenfeld A, Bar Y, Merlob P, Ovadia Y. NSAIDS: maternal and fetal considerations. Am J Reprod Immunol 1992; 3-4: 14 I -7.

2. Barton JR, Sibai BM. Low-dose aspirin to improve perinatal outcome. Clin Obstet Gynecol 199I; 34:25I-6I.

3. Ungváry GY, Tátrai E, Lórincz M, Barcza GY. Combined embryotoxic action of toluene, a widely used industrial chemical, and acetylsalicylic acid (aspirin). Teratology 1983; 27:26I9.

4. Kimmel CA, Wilson JG, Schumacher HJ Studies on metabolism and identification of the causative agent in aspirin teratogenesis in rats. Teratology I 97 I; 4: 15-24.

5. Koshaskji RP, Schulert AR. Biochemical and mechanisms of salicylate teratology in the rat. Biochem Pharmacol 1 973; 22:407.

6. EspiridiãoS, Ajeje R, Kopelman BI, Simões MJ, Evêncio-NetoJ, Kulay-Júnior L. Ação crônica doácido acetilsalicílico na prenhez da rata. Rev Bras Ginecol Obstet 1998; 20:245-9.

7. Macedo PHG. Estudo morfológico de fetose placentas de ratas sob a ação do etanol e/ou ácido acetilsalicílico (tese). São Paulo: Universidade Federal de São Paulo, Escola Paulista de Medicina; 1998.

8. Fonseca TT. Bajas dosis de aspirina en la prevención del retardo de crecimiento intrauterino en el embarazo de riesgo (dissertação). Nicaragua: Universidade Nacional Autônoma de Nicaragua; 1999.

9. Giavini E, Lemonica IP, Lou Y, Broccia ML, Prati $M$. Induction of micronuclei and toxic effects in embryos of pregnant rats treated before implantation with anticancer drugs: cyclophosphamide, cis-platinum, adriamycin. Teratog Carcinog Mutagen 1990; 10:4 1 7-26.

10. Damasceno DC, Lemonica IP. Embryotoxicity and anti-implantation effects of rosemary (Rosmarinus officinalis L.) extract in pregnant rats within preimplantation period. Rev Bras Toxicol 1999; 12:47-54.

I I. Calderon IMP. Modelo experimental em ratas para estudo do binômio diabete e gravidez (dissertação). Botucatu: Universidade Estadual Paulista; 1988.

12. Salewski E. Farbemethode zum markroskopishen nachweis von implantatconsstellen am uterus der ratter naunyn schmuderbergs. Arch Pharm (Weinheim) 1964; 247:367.

13. Lemonica IP, Damasceno DC, Di Stasi LC. Study of the embryotoxic effects of an extract of rosemary (Rosmarinus officinalis L.). Braz J Med Biol Res 1996; 29:223-7.

14. Wilson JG. Methods for administering agents and detecting malformations in experimental animal. In: Wilson JG, Warkany J. Teratology: principle and techniques. Chicago: University Chicago Press; 1965. p.262-77.

I5. Staples RE, Schnell VL. Refinements in rapid clearing technic in the $\mathrm{KOH}$-alizarin red $\mathrm{S}$ method for fetal bone. Stain Technol 1964; 39:6I-3.

I6. Aliverti V, Bonanomi L, Giavini E, Leone VG, Mariani L. The extent of fetal ossification as an index of delayed development in teratogenic studies on the rat. Teratology 1979; 20:23742.

17. Siegel S. Estatística não-paramétrica (para ciências do comportamento). São Paulo: Mc Graw-Hill; 1975.

18. Berquó ES, Souza JMP, Gotlick SLD. Bioestatística. São Paulo: E.P.U.; I98I.

19. Pederson AK, Fitzgerald GA. Dose-related kinetics of aspirin, presystemic acetylation of platelet cyclooxigenase. N Engl J Med 1984; 311:1206.

20. Nishimura $H$, Tanimura T. Clinical aspects of the teratogenicity of drugs. New York: American Elsevier Publishing Company; 1976. p.204- 10.

21. Schardein JL. Drugs as teratogens. Cleveland: CRF Press Inc; 1976. p.57-60.

22. Streissguth AP, Treder RP, Barr HM, Shepard TH, Bleyer WA, Sampson PD, et al. Aspirin and acetaminophen use by pregnancy woman and subsequent child $\mathrm{IQ}$ and attention decrements. Teratology 1987; 35:2 I I-9.

23. Wichramaratne GA. The post-natal fate of supranumerary ribs in rat teratogenicity studies. J Appl Toxicol 1988; 8:9 I-4.

24. Joschko M, Dreosti IE, Tulsi RS. The teratogenic effects of salicylic acid on the developing nervous system in rats in vitro. Teratology 1993; 48: $105-14$

25. Ying Y, Cai YX, Lou YJ. Effects of blastocyst deficiencies induced by aspirin treatment during preimplantation period in rats on development of embryos after implantation. Yoaxue Xuebao 1996; 31:416-9.

26. Lou YJ, Ding GS, Tu ZH. Toxicity to transferred rat embryos after aspirin treatment during preimplantation stage in vivo. Acta Pharmacol Sin 1996; 17:52-4

27. Lemonica IP. Embriofetotoxicidade. In: Oga S Fundamentos de toxicologia. Rio de Janeiro: Guanabara-Koogan; 1996. p.86-94.

28. Beguin EP. Rinón y medicamentos. Bol Hosp San Juan 1996; 43:2 I 5-7.

29. Taylor P. Practical teratology. New York: Academic Press; 1986. p.50-7.

30. Lemonica IP; Alvarenga CMD. Abortive and teratogenic effect of Acanthospermum hispidum DC. and Cajanus cajan (L.) Millps. in pregnant rats. J Etnopharmacol 1994; 43:39-44.

Artigo recebido: 20/04/200I Aceito para publicação: | |/|2/200 | 